OPEN ACCESS

Edited by:

Vidya Gopalakrishnan, University of Texas MD Anderson Cancer Center, United States

Reviewed by: Wafik Zaky,

University of Texas MD Anderson Cancer Center, United States Mariko D. DeWire, Cincinnati Children's Hospital Medical Center, United States

${ }^{*}$ Correspondence: Quan Cheng

chengquan@csu.edu.cn Zhixiong Liu

zhixiongliu@csu.edu.cn

Specialty section:

This article was submitted to Neuro-Oncology and Neurosurgical

Oncology,

a section of the journal Frontiers in Oncology

Received: 14 July 2019 Accepted: 05 March 2020 Published: 09 April 2020

Citation:

Liu Z, Feng S, Li J, Cao H, Huang J, Fan F, Cheng L, Liu Z and Cheng $Q$ (2020) The Epidemiological Characteristics and Prognostic

Factors of Low-Grade Brainstem Glioma: A Real-World Study of Pediatric and Adult Patients. Front. Oncol. 10:391. doi: 10.3389/fonc.2020.00391

\section{The Epidemiological Characteristics and Prognostic Factors of Low-Grade Brainstem Glioma: A Real-World Study of Pediatric and Adult Patients}

\author{
Zhuoyi Liu ${ }^{1,2}$, Songshan Feng ${ }^{1,2}$, Jing Li $^{3}$, Hui Cao ${ }^{4}$, Jun Huang ${ }^{1,2}$, Fan Fan ${ }^{1,2,5}$, Li Cheng ${ }^{6}$, \\ Zhixiong Liu ${ }^{1,2 *}$ and Quan Cheng ${ }^{1,2,7 *}$ \\ ${ }^{1}$ Department of Neurosurgery, Xiangya Hospital, Center South University, Changsha, China, ${ }^{2}$ National Clinical Research \\ Center for Geriatric Disorders, Xiangya Hospital, Central South University, Changsha, China, ${ }^{3}$ Department of Rehabilitation, \\ Second Xiangya Hospital, Central South University, Changsha, China, ${ }^{4}$ Department of Psychiatry, The Second People's \\ Hospital of Hunan Province, The Hospital of Hunan University of Chinese Medicine, Changsha, China, ${ }^{5}$ Center for Medical \\ Genetics and Hunan Provincial Key Laboratory of Medical Genetics, School of Life Sciences, Central South University, \\ Changsha, China, ${ }^{6}$ Department of Emergency, Fengyang County Hospital of Traditional Chinese Medicine, Fengyang, China, \\ ${ }^{7}$ Department of Clinical Pharmacology, Xiangya Hospital, Central South University, Changsha, China
}

Purpose: Our current understanding of low-grade brainstem glioma (LGBSG) is still limited. This study aimed to conduct a large-scale population-based real-world study to understand the epidemiological characteristics of LGBSG and determine the predictive factors of cancer-specific survival (CSS) and overall survival (OS) of LGBSG patients.

Patients and Methods: We used Surveillance Epidemiology and End Results database to conduct this study of patients with histologically confirmed LGBSG. Patient demographics, tumor characteristics, and treatment options were compared between pediatric and adult patients. Univariate and multivariate analyses were employed to determine prognostic factors of CSS and OS. Kaplan-Meier curve and decision tree were used to confirm the prognostic factors. All variables were further identified by L1-penalized (Lasso) regression and then a nomogram was established to predict the 5- and 8-year CSS and OS rate. The precision of the nomogram was evaluated by calibration plots, Harrell's concordance index, and time-dependent receiver operating characteristic curve. The clinical use of nomogram was estimated by decision curve analysis.

Results: A cohort of 305 patients with LGBSG, including 165 pediatric and 140 adult patients, was analyzed. Adult and pediatric patients showed different patterns concerning tumor size, tumor extension, adjuvant therapy, and survival rate. Univariate analysis revealed that pediatric group, gross total resection (GTR), World Health Organization grade II, radiotherapy, extension to ventricular system, and diffuse astrocytic and oligodendroglial tumor (DAOT) were significantly associated with CSS. Multivariate analysis showed that pediatric group, metastasis, ventricular system involvement, and DAOT were independently associated with CSS. The prognostic factors were further confirmed by Kaplan-Meier curve and decision tree. Kaplan-Meier curve also showed 
that adjuvant therapy added no benefits in patients with GTR and non-GTR. In addition, the nomogram was developed and the C-index of internal validation for CSS was 0.87 (95\% Cl, 0.78-0.96).

Conclusion: This study shows that pediatric and adult patients have different tumor characteristics, treatment options, and survival rate. Pediatric group, DAOT, ventricular system involvement, and metastasis were identified as independent prognostic factors for CSS by multivariate analysis. Adjuvant therapy showed no benefits on CSS in patients with GTR and non-GTR. The nomogram was discriminative and clinically useful.

Keywords: low-grade brainstem glioma, SEER, cancer-specific survival, overall survival, nomogram, real-world study

\section{INTRODUCTION}

Brainstem glioma (BSG) encompasses a heterogeneous group of tumors, which are classified according to epidemiological, imaging [magnetic resonance imaging (MRI)], and pathological characteristics. Epidemiologically, BSG accounts for $4.3 \%$ of all gliomas as recorded in the most recent Central Brain Tumor Registry of the United States (CBTRUS) report (1). Notably, BSG constitutes $\sim 15 \%$ of pediatric brain tumors and $<2 \%$ of adult gliomas $(1,2)$. Based on MRI characteristics and surgical experience, Choux et al. (3) classified BSG into types of diffuse, intrinsic focal, extrinsic focal, and cervicomedullary, and this remains to be most recent and widely accepted categorization system of BSG. Diffuse brainstem pontine glioma (DIPG) is associated with dismal prognosis in both pediatric and adult patients, being highly infiltrative and less amenable to surgery $(4,5)$. Pathologically, pediatric and adult patients with highgrade BSG (HGBSG), including World Health Organization (WHO) grades III and IV BSG, have worse clinical outcomes $(6,7)$. Over the last two decades, we have gained deep understanding on pediatric DIPG and HGBSG in terms of biological characteristics, prognostic factors, and treatment strategies (8-11). However, little is known about low-grade BSG (LGBSG) especially its presentation in adult patients. Only few single-center retrospective studies with small population concerning pediatric LGBSG were available. And no study focusing on adult LGBSG has been published to date. Regarding its treatment modalities, surgical resection has improved with advancing imaging and neurosurgery techniques (12-14). At the same time, efforts were devoted to investigate the adjuvant therapy including radiotherapy (RT) and chemotherapy (CT) for LGBSG patients (15-19). However, there is no consensus on the benefits of surgical resection and adjuvant therapy for LGBSG.

This population-based real-world study was conducted to address this challenge. A search was performed on the SEER (Surveillance Epidemiology and End Results) database, which identified 165 pediatric and 140 adult patients with histologically confirmed LGBSG from 2004 to 2015. The major purpose of this study was to determine the prognostic factors influencing cancerspecific survival (CSS) and overall survival (OS), which could help to optimize the management of patients with LGBSG.

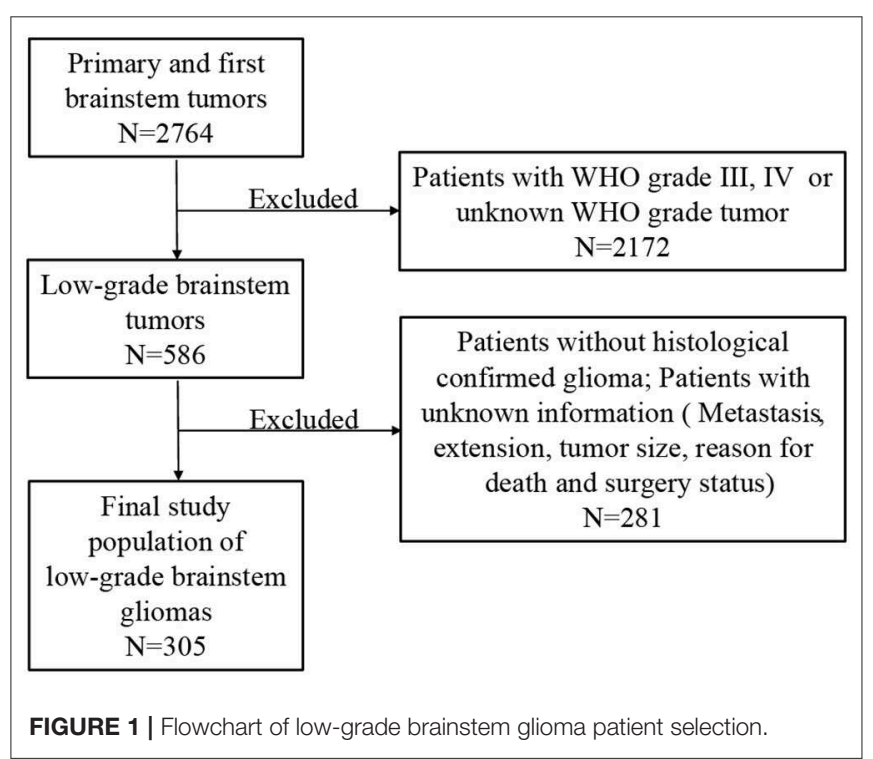

\section{METHODS}

\section{Study Population}

The SEER database, which is maintained by the National Cancer Institute, was searched to identify data deposited between 2004 and 2015. The SEER database provides prospectively collected data on patients with deidentified information. For this reason, no approval was required from the institutional review board for this study. All patients with first and primary brainstem tumor were included. Patients with WHO grade III or IV or unknown WHO grade tumors were excluded. Patients without histologically confirmed glioma and other crucial variates (metastasis, extension, tumor size, reason for death, and surgery status) were also excluded (Figure 1).

\section{Covariates Included}

The following patient data were obtained for the analysis: age at diagnosis (patients aged $<22$ years were assigned to the pediatric group, and those aged $\geq 22$ years were assigned to adult group), sex, race (white, black, American Indian/Alaska Native, Asian/Pacific Islander), marital 
status (married, unmarried), WHO grade (I, II), surgery [unknown surgery status, local excision/biopsy, subtotal resection, gross total resection (GTR)], tumor size (size $\leq 3.6 \mathrm{~cm}$, size $>3.6 \mathrm{~cm}$, the best cutoff value was defined according to X-tile software), metastasis (yes, no), RT and CT (both, none/unknown, RT, CT), and extension pattern (brainstem, cerebellum, ventricular, and other categories). We divided the histological type into diffuse astrocytic and oligodendroglial tumors (DAOTs), other astrocyte tumors (OATs), and ependymal tumors (ETs) according to the 2016 WHO classification of tumors of the central nervous system (Table 1).

\section{Statistical Analyses}

To analyze all different prognostic variables associated with the CSS and OS, both univariate and multivariate cox proportional hazard models were applied to calculate hazard ratios (HRs) and the corresponding 95\% confidence intervals (CIs). $P<$ 0.05 was considered statistically significant. And Kaplan-Meier curves and decision tree were plotted to compare the CSS of patients with LGBSG patients by all different prognostic factors. The study population was randomly divided into training group $(n=152)$ and test group $(n=153)$. All different variables were further identified by L1-penalized (Lasso) regression model. The risk scores were then calculated according to the formula, risk score $=\beta 1 \mathrm{X} 1+\beta 2 \mathrm{X} 2+\ldots+$ $\beta \mathrm{nXn}$ ( $\beta$, regression coefficient; $\mathrm{X}$, prognostic factors). Then a nomogram was developed using the package of RMS in $\mathrm{R}$ version 3.5.1 (http://www.r-project.org/). A calibration curve was used for internal validation, which described the average predictive value against actual observation and evaluated the performance of nomogram visually. Harrell's concordance index (C-index) and time-dependent receiver operating characteristic (ROC) curve were used to evaluate the discrimination of nomogram to assess the consistency between the actual and predicted CSS rate. The clinical use of nomogram was estimated by decision curve analysis (DCA), which is a novel method that estimates predictive models from the perspective of clinical consequences.

\section{RESULTS}

\section{Patient Population and Baseline Characteristics}

A total of 305 LGBSG patients were analyzed. Among them, 165 were pediatric with a mean age of 8.31 years $(<22$ years), and 140 were adult with a mean age of 46.01 years (22-85 years). The data showed that LGBSG had a slight male preponderance $(56.7 \%)$, but it was not statistically significant $(P=0.500)$. At the time of data collection, the CSS rates for pediatric and adults patients were 93.9 and $86.4 \%$, respectively $(P=0.042)$. Majority of patients were white $(n=261,85.5 \%)$, whereas $27(8.9 \%)$ were black, and 17 (5.6\%) were Asian/Pacific Islander. The majority of patients were diagnosed during 2010-2015 compared to 2004-2009 ( $n$ $=171,56.7 \%$ vs. $n=134,43.9 \%)$. At the time of data
TABLE 1 | Patient demographics, tumor characteristics, and treatment options of 305 low-grade brainstem glioma patients.

\begin{tabular}{|c|c|c|c|c|}
\hline Characteristic & $\begin{array}{l}\text { ALL } \\
n(\%)\end{array}$ & $\begin{array}{c}\text { Pediatric } \\
n(\%)\end{array}$ & $\begin{array}{l}\text { Adult } \\
n(\%)\end{array}$ & $P$ \\
\hline Population size & $305(100)$ & $165(54.1)$ & $140(45.9)$ & \\
\hline Age, median (range), y & & $8.31(<22)$ & $46.01(22-85)$ & \\
\hline Era of diagnosis & & & & 0.353 \\
\hline 2004-2009 & $134(43.9)$ & 77 (25.2) & $57(18.7)$ & \\
\hline 2010-2015 & $171(56.1)$ & 88 (28.9) & $83(27.2)$ & \\
\hline Sex & & & & 0.500 \\
\hline Female & 132 (42.3) & 68 (22.3) & $64(20.0)$ & \\
\hline Male & $173(56.7)$ & 97 (31.8) & $76(24.9)$ & \\
\hline Race & & & & 0.565 \\
\hline Asian/Pacific Islander & $17(5.6)$ & $9(3.0)$ & $8(2.6)$ & \\
\hline Black & 27 (8.9) & $12(3.9)$ & $15(5.0)$ & \\
\hline White & $261(85.5)$ & $144(47.2)$ & 117 (38.3) & \\
\hline \multicolumn{5}{|l|}{ Marital status } \\
\hline Unmarried & $224(73.4)$ & 164 (53.8) & $60(19.6)$ & $<0.001^{\dagger}$ \\
\hline Married & 81 (26.6) & $1(0.3)$ & 80 (26.3) & \\
\hline \multicolumn{5}{|l|}{ WHO grade } \\
\hline I & 137 (44.9) & $98(32.1)$ & 39 (12.8) & $<0.001^{\dagger}$ \\
\hline$\|$ & $168(55.1)$ & $67(22.0)$ & $101(33.1)$ & \\
\hline Surgery & & & & 0.988 \\
\hline Local excision/biopsy & $60(19.7)$ & 32 (10.5) & $28(9.2)$ & \\
\hline STR & 65 (21.3) & 35 (11.5) & $30(9.8)$ & \\
\hline GTR & $180(59.0)$ & $98(32.1)$ & $82(26.9)$ & \\
\hline \multicolumn{5}{|l|}{ Size } \\
\hline$\leq 3.6 \mathrm{~cm}$ & 158 (51.8) & 66 (21.6) & $92(30.2)$ & $<0.001^{\dagger}$ \\
\hline$>3.6 \mathrm{~cm}$ & 147 (48.2) & 99 (32.5) & $48(15.7)$ & \\
\hline \multicolumn{5}{|l|}{ Metastasis } \\
\hline Yes & $8(2.6)$ & $4(1.3)$ & $4(1.3)$ & 1.000 \\
\hline No & 297 (97.4) & $161(52.8)$ & 136 (46.6) & \\
\hline \multicolumn{5}{|l|}{ Adjuvant therapy } \\
\hline Both & $18(5.9)$ & $14(4.6)$ & $4(1.3)$ & $<0.001^{\dagger}$ \\
\hline Radiotherapy & 100 (32.8) & $42(13.7)$ & $58(19.1)$ & \\
\hline Chemotherapy & $25(8.2)$ & $24(7.9)$ & $1(0.3)$ & \\
\hline None/unknown & $162(53.1)$ & $85(27.9)$ & 77 (25.2) & \\
\hline \multicolumn{5}{|l|}{ Extension } \\
\hline Brainstem & 120 (39.4) & 75 (24.6) & $45(14.8)$ & $<0.001^{\dagger}$ \\
\hline Cerebellum & $25(8.2)$ & $15(4.9)$ & $10(3.3)$ & \\
\hline Ventricular & $105(34.4)$ & $43(14.1)$ & $62(20.3)$ & \\
\hline Other & $55(18.0)$ & $32(10.5)$ & $23(7.5)$ & \\
\hline \multicolumn{5}{|c|}{ Cancer-specific death status } \\
\hline Alive & $276(90.5)$ & 155 (50.8) & $121(39.7)$ & $0.042^{\dagger}$ \\
\hline Dead & $29(9.5)$ & $10(3.3)$ & $19(6.2)$ & \\
\hline \multicolumn{5}{|l|}{ Vital status } \\
\hline Alive & 265 (86.8) & 152 (49.8) & $113(37.0)$ & $0.006^{\dagger}$ \\
\hline Dead & 40 (13.2) & $13(4.3)$ & 27 (8.9) & \\
\hline \multicolumn{5}{|l|}{ Histology } \\
\hline DAOT & $31(10.2)$ & $19(6.2)$ & $12(4.0)$ & $<0.001^{\dagger}$ \\
\hline ET & 138 (45.2) & $45(14.8)$ & $93(30.4)$ & \\
\hline OAT & $136(44.6)$ & $101(33.1)$ & 35 (11.5) & \\
\hline
\end{tabular}

${ }^{t} P<0.05$, statistically significant. 
TABLE 2 | Univariate and multivariate Cox proportional hazard regression analyses to determine prognostic factors of cancer-specific survival for patients with low-grade brainstem glioma.

\begin{tabular}{|c|c|c|c|c|c|c|}
\hline & \multirow[t]{2}{*}{ 5-y CSS rate (\%) } & \multirow[t]{2}{*}{$10-y$ CSS rate (\%) } & \multicolumn{2}{|c|}{ Univariate analysis } & \multicolumn{2}{|c|}{ Multivariate analysis } \\
\hline & & & HR (95\% Cl) & $P$ & HR (95\% Cl) & $P$ \\
\hline \multicolumn{7}{|l|}{ Age } \\
\hline$\geq 22$ Adult & 84.9 & 74.7 & 1 [Reference] & & 1 [Reference] & \\
\hline$<22$ Pediatric & 93.2 & 86.6 & $0.39(0.18-0.84)$ & $0.016^{\dagger}$ & $0.28(0.10-0.76)$ & $0.012^{\dagger}$ \\
\hline \multicolumn{7}{|l|}{ Sex } \\
\hline Female & 88.1 & 83.5 & 1 [Reference] & & 1 [Reference] & \\
\hline Male & 90.7 & 90.7 & $0.72(0.35-1.49)$ & 0.375 & $0.96(0.42-2.17)$ & 0.918 \\
\hline \multicolumn{7}{|l|}{ Race } \\
\hline White & 90.0 & 7.6 & 1 [Reference] & & 1 [Reference] & \\
\hline Black & 83.5 & 83.5 & $1.62(0.56-4.66)$ & 0.374 & $1.42(0.46-4.43)$ & 0.545 \\
\hline Asian/Pacific Islander & 90.0 & / & $0.73(0.10-5.37)$ & 0.753 & $1.41(0.18-11.22)$ & 0.744 \\
\hline \multicolumn{7}{|l|}{ Marital status } \\
\hline Unmarried & 88.1 & 93.6 & 1 [Reference] & & 1 [Reference] & \\
\hline Married & 85.0 & 86.2 & $1.02(0.45-2.31)$ & 0.956 & $0.54(0.20-1.46)$ & 0.226 \\
\hline \multicolumn{7}{|l|}{ Grade } \\
\hline 1 & 93.6 & 93.6 & 1 [Reference] & & 1 [Reference] & \\
\hline$\|$ & 86.2 & 82.8 & $2.69(1.15-6.30)$ & $<0.001^{\dagger}$ & $2.57(0.30-21.75)$ & 0.386 \\
\hline \multicolumn{7}{|l|}{ Surgery } \\
\hline Local excision/biopsy & 81.3 & 81.3 & 1 [Reference] & & 1 [Reference] & \\
\hline STR & 79.1 & / & $1.22(0.50-2.97)$ & 0.659 & $1.64(0.61-4.40)$ & 0.570 \\
\hline GTR & 95.5 & 93.1 & $0.28(0.11-0.69)$ & $0.005^{\dagger}$ & $0.40(0.15-1.12)$ & 0.081 \\
\hline \multicolumn{7}{|l|}{ Size } \\
\hline$\leq 3.6 \mathrm{~cm}$ & 88.4 & 86.2 & 1 [Reference] & & 1 [Reference] & \\
\hline$>3.6 \mathrm{~cm}$ & 90.6 & 88.9 & $0.82(0.39-1.70)$ & 0.591 & $1.26(0.49-3.25)$ & 0.634 \\
\hline \multicolumn{7}{|l|}{ Metastasis } \\
\hline No & 90.0 & 87.8 & 1 [Reference] & & 1 [Reference] & \\
\hline Yes & 68.6 & / & $2.75(0.65-11.57)$ & 0.168 & 5.20 (1.03-26.38) & $0.046^{\dagger}$ \\
\hline \multicolumn{7}{|l|}{ Adjuvant therapy } \\
\hline None/unknown & 93.9 & 92.0 & 1 [Reference] & & 1 [Reference] & \\
\hline Radiotherapy & 81.6 & 81.6 & $2.46(0.46-9.71)$ & $0.028^{\dagger}$ & $1.48(0.57-3.82)$ & 0.421 \\
\hline Chemotherapy & 94.4 & 78.7 & $1.37(0.30-6.27)$ & 0.683 & $0.81(0.14-4.50)$ & 0.806 \\
\hline Both & 86.2 & / & $2.12(0.46-9.71)$ & 0.333 & $0.93(0.16-5.36)$ & 0.938 \\
\hline \multicolumn{7}{|l|}{ Extension } \\
\hline Brainstem & 85.0 & 83.0 & 1 [Reference] & & 1 [Reference] & \\
\hline Cerebellum & 92.0 & 92.0 & $0.58(0.13-2.51)$ & 0.462 & $0.64(0.13-3.16)$ & 0.581 \\
\hline Ventricular & 95.4 & 92.2 & $0.36(0.13-0.99)$ & $0.049^{\dagger}$ & $0.40(0.14-1.20)$ & $0.010^{\dagger}$ \\
\hline Other & 87.4 & 87.4 & $0.85(0.33-2.16)$ & 0.727 & $0.91(0.30-2.83)$ & 0.877 \\
\hline \multicolumn{7}{|l|}{ Histology } \\
\hline ET & 92.1 & 88.2 & 1 [Reference] & & 1 [Reference] & \\
\hline OAT & 93.6 & 93.6 & $0.64(0.25-1.65)$ & 0.358 & $1.83(0.20-17.33)$ & 0.596 \\
\hline DAOT & 59.1 & 59.1 & $5.65(2.45-13.08)$ & $<0.001^{\dagger}$ & $5.14(1.72-15.39)$ & $0.003^{\dagger}$ \\
\hline
\end{tabular}

${ }^{\dagger} P<0.05$, statistically significant.

collection, 265 patients (86.8\%) were alive, whereas 40 cases (13.2\%) had died. Of the 40 deaths, 29 died of cancerspecific events.

Metastasis occurred in eight patients (2.6\%), including four pediatric and four adult patients, indicating no difference $(P=$ 1.000). In contrast, there were significant differences between pediatric and adult patients in tumor size; the proportion of patients with tumor size $>3.6 \mathrm{~cm}$ in the adult groups was significantly lower than that in pediatric patients (34.3 vs. $60.0 \%, P<0.001)$. In terms of tumor extension pattern, it showed significant difference between the two groups $(P<$ $0.001)$; the proportion of patients with tumor extended to 


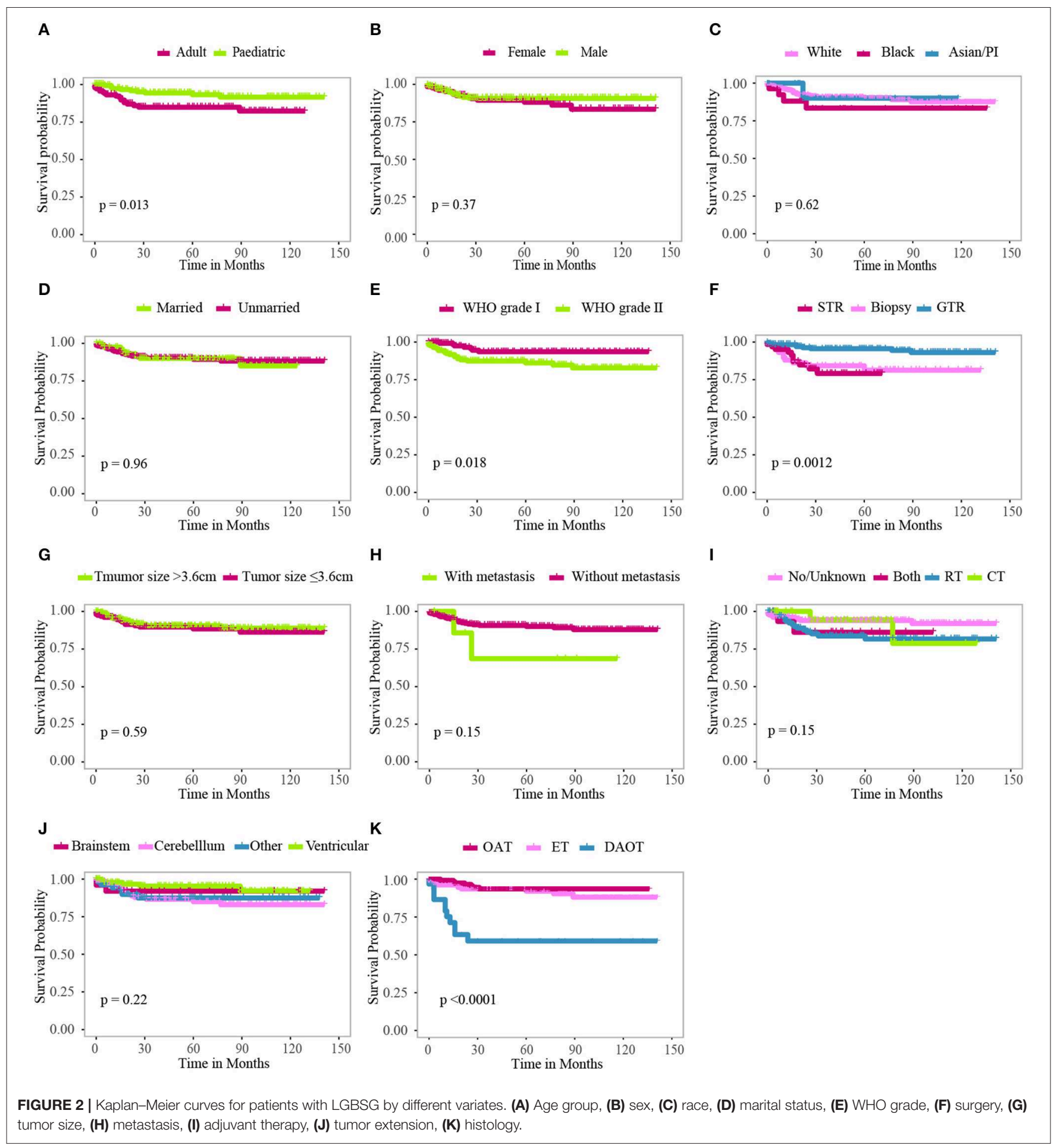

ventricular system in adults was significantly higher than that in pediatric patients ( 44.3 vs. $26.1 \%, P<0.001$ ). Concerning treatment options, the extent of surgery was not significantly different between the two groups $(P=0.988)$. But for adjuvant therapy, notable differences were observed between the two groups; more adult patients received RT (41.4 vs. 25.5\%), and fewer adult patients received CT $(0.1$ vs. $14.5 \%)(P<0.001)$ (Table 1). 

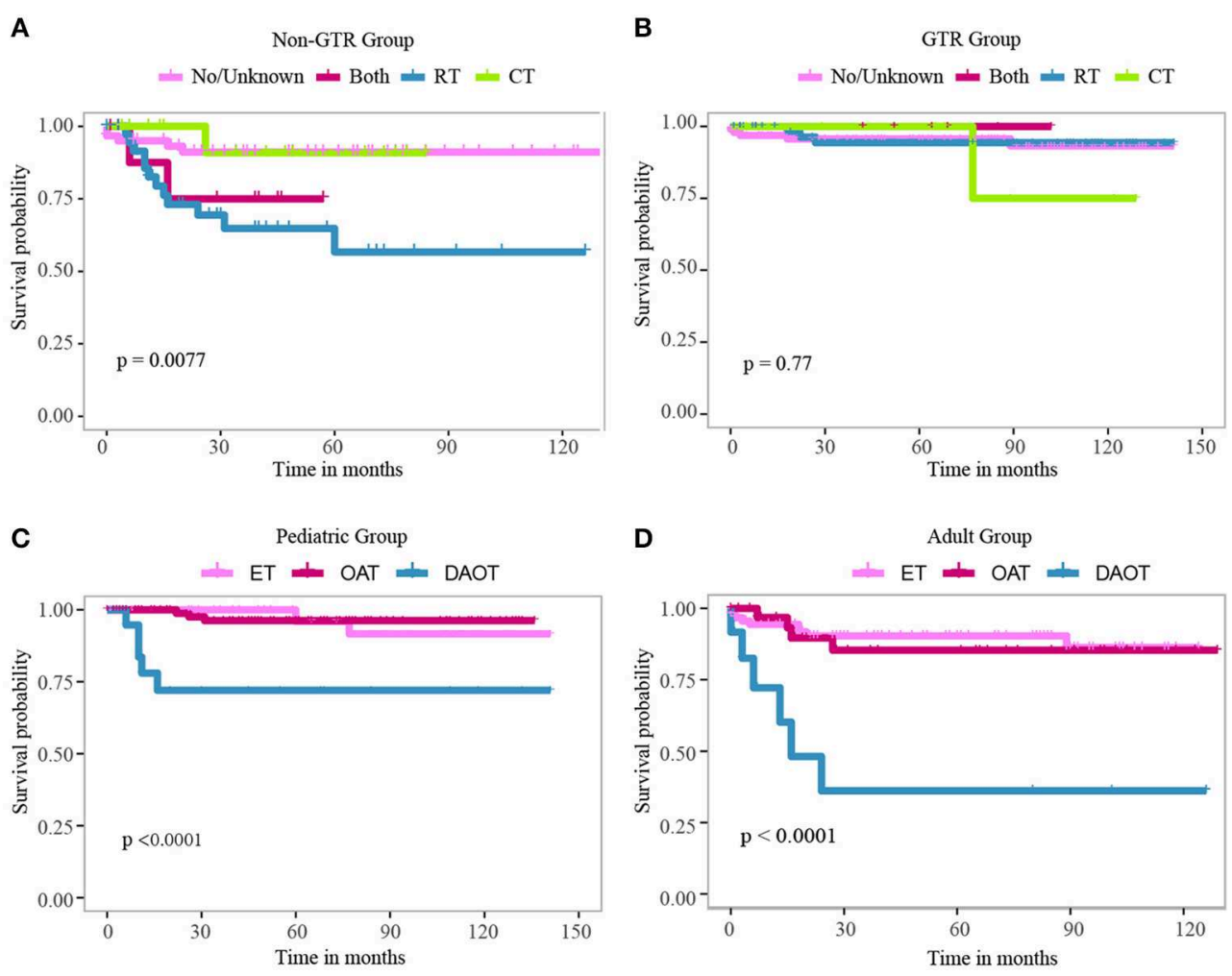

FIGURE 3 | Kaplan-Meier curve for patients with LGBSG in non-GTR group (A) and GTR group (B) treated with different adjuvant therapies. Kaplan-Meier curve for pediatric patients (C) and adult patients (D) with different histology types.

\section{Prognostic Factors of CSS and OS}

The univariate analysis showed that the pediatric group (HR, 0.39 ; 95\% CI, $0.18-0.84 ; P=0.016$ ), GTR (HR, $0.28 ; 95 \%$ CI, $0.11-0.69 ; P=0.005$ ), and extension to ventricular system (HR, 0.36; 95\% CI, 0.13-0.99; $P=0.049$ ) were associated with increased CSS rate $(P<0.05)$. In contrast, WHO grade II (HR, $2.69(1.15-6.30 P<0.001)$, DAOT (HR, 5.65; 95\% CI, $2.45-13.08 ; P<0.001$ ), and RT (HR, 2.46; 95\% CI, 0.469.71; $P=0.028)$ were significantly associated with decreased CSS rate $(P<0.05)$. In multivariate analysis, pediatric group (HR, $0.28 ; 95 \% \mathrm{CI}, 0.10-0.76 ; P=0.012$ ) and ventricular system involvement (HR, 0.40; 95\% CI, $0.14-1.20 ; P=0.010)$ were independently associated with improved CSS rate. On the contrary, metastasis (HR, 5.20; 95\% CI, 1.03-26.38; $P=$ 0.046 ) and DAOT (HR, 5.14; 95\% CI, 1.72-15.39; $P=0.003$ ) were independently associated with decreased CSS rate. Analysis of surgical procedures showed that GTR ( $\mathrm{HR}, 0.40 ; 95 \% \mathrm{CI}$, $0.15-1.12 ; P=0.081$ ) was not significantly associated with better CSS after adjusting for confounding effects of each variable when compared with biopsy group. World Health Organization grade II (HR, 2.57; 95\% CI, $0.30-21.75 ; P=$ 0.386 ) also lost its significance in multivariate analysis (Table 2). The predictive factors of OS were similar, which were only slightly different from those of CSS in $\mathrm{HR}, 95 \% \mathrm{CI}$, and $P$-value (Table S1).

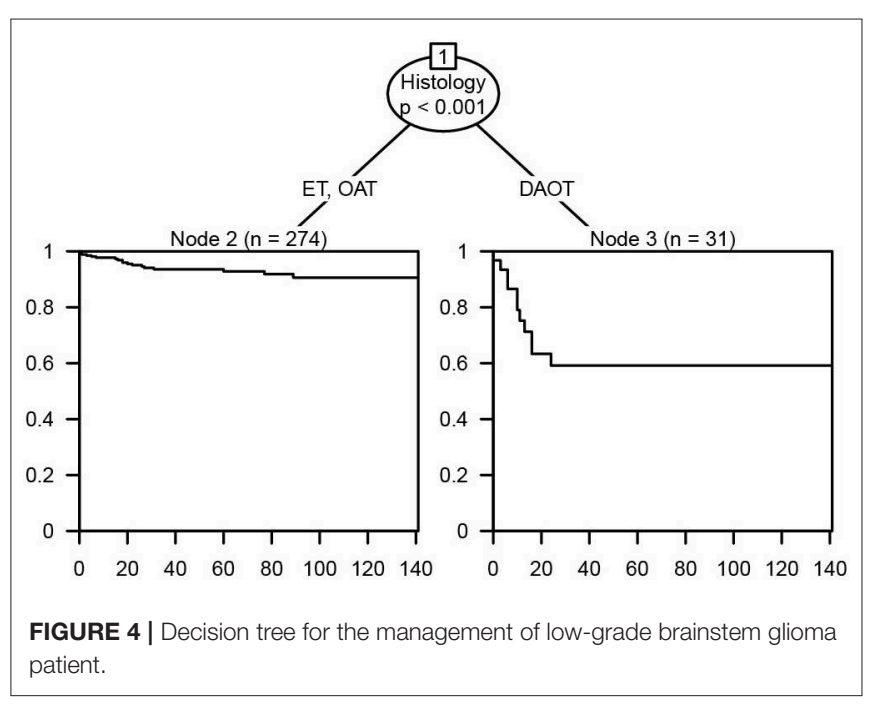

Kaplan-Meier curves were plotted to compare the CSS of LGBSG patients by different variates. The result showed that age group $(P=0.013)$, WHO grade $(P=0.018)$, surgery $(P=0.0012)$, and histology $(P<0.0001)$ showed significant difference (Figure 2). To assess the benefit of adjuvant therapy 
TABLE 3 | Training and validation cohort for nomogram to predict 5- and 8-year cancer-specific survival rates of low-grade brainstem glioma patient.

\begin{tabular}{|c|c|c|c|}
\hline & Training $n(\%)$ & Validation $n(\%)$ & $P$ \\
\hline Age & & & 1.000 \\
\hline$\geq 22$ Adult & $70(23.0)$ & $70(23.0)$ & \\
\hline$<22$ Pediatric & $82(26.9)$ & $83(27.1)$ & \\
\hline Sex & & & 0.448 \\
\hline Female & $62(20.3)$ & $70(23.0)$ & \\
\hline Male & $90(29.5)$ & $83(27.2)$ & \\
\hline Race & & & 0.199 \\
\hline White & $126(41.3)$ & $135(44.3)$ & \\
\hline Black & $14(4.6)$ & $13(4.3)$ & \\
\hline Asian or Pacific Islander & $12(3.9)$ & $5(1.6)$ & \\
\hline Marital status & & & 0.417 \\
\hline Unmarried & $108(35.4)$ & $116(38.0)$ & \\
\hline Married & $44(14.4)$ & $37(12.2)$ & \\
\hline WHO grade & & & 0.272 \\
\hline 1 & $63(20.7)$ & $74(24.3)$ & \\
\hline$\|$ & $89(29.2)$ & $79(25.8)$ & \\
\hline Surgery & & & 0.399 \\
\hline Local excision/biopsy & $34(11.2)$ & $26(8.5)$ & \\
\hline STR & $29(9.5)$ & $36(11.8)$ & \\
\hline GTR & $89(29.2)$ & $91(29.8)$ & \\
\hline Size & & & 0.862 \\
\hline$\leq 3.6 \mathrm{~cm}$ & $80(26.2)$ & $78(25.6)$ & \\
\hline$>3.6 \mathrm{~cm}$ & $72(23.6)$ & $75(24.6)$ & \\
\hline Metastasis & & & 0.287 \\
\hline No & $150(49.2)$ & $147(48.2)$ & \\
\hline Yes & $2(0.6)$ & $6(2.0)$ & \\
\hline Adjuvant therapy & & & 0.920 \\
\hline No/unknown & $83(27.2)$ & 79 (25.9) & \\
\hline Both & $9(3.0)$ & $9(3.0)$ & \\
\hline Radiotherapy & $49(16.0)$ & $51(16.7)$ & \\
\hline Chemotherapy & $11(3.6)$ & $14(4.6)$ & \\
\hline Extension & & & 0.731 \\
\hline Brainstem & $61(22.0)$ & 59 (19.3) & \\
\hline Cerebellum & $14(4.6)$ & $11(3.6)$ & \\
\hline Ventricular & $53(17.4)$ & $52(17.0)$ & \\
\hline Other & $24(7.9)$ & $31(10.2)$ & \\
\hline Cancer-specific death event & & & 1 \\
\hline Alive & $138(45.3)$ & $138(45.2)$ & \\
\hline Dead & $14(4.6)$ & $15(4.9)$ & \\
\hline Vital status & & & 0.595 \\
\hline Alive & $130(42.6)$ & 135 (44.3) & \\
\hline Dead & $22(7.2)$ & $18(5.9)$ & \\
\hline
\end{tabular}

in the non-GTR group, one additional Kaplan-Meier curve was plotted. The result showed that patients who received RT or RT combined with CT had worse survival $(P=0.0077)$ (Figure 3). The decision tree identified that histological-type DAOT $(P<$ 0.001 ) was the most distinguishable factor for survival period (Figure 4).

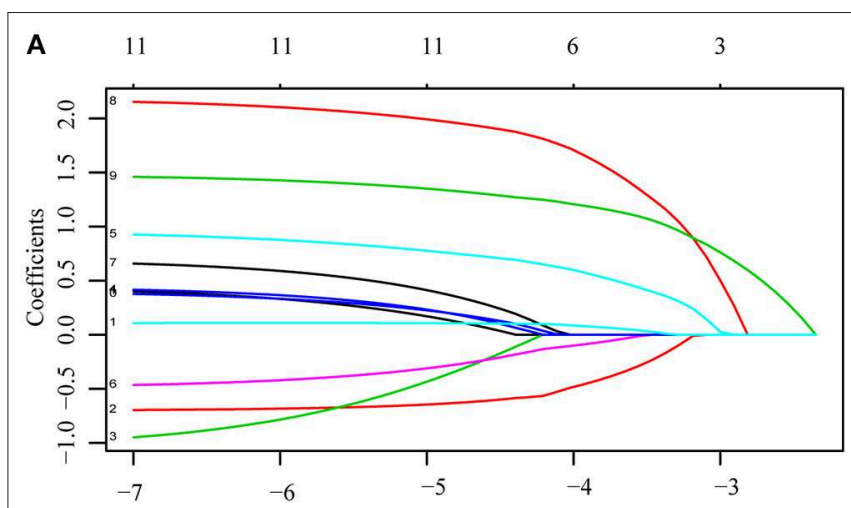

B

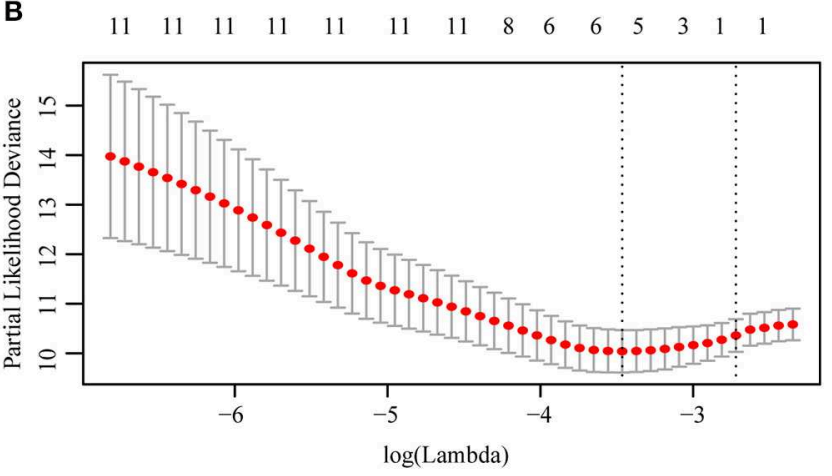

FIGURE 5 | L1-penalized (Lasso) regression model were applied to further identified prognostic factors in training cohort. Race, surgery, histology, and adjuvant therapy were identified for CSS (A). LASSO coefficient profiles of the features (B). Ten-time cross-validation for tuning paremeter selection in the Lasso Model.

\section{Prognostic Nomogram for CSS and OS}

The population was randomly divided into training group ( $n=$ $152)$ and validation group $(n=153)$ (Table 3$)$. We applied L1penalized (Lasso) regression model to further identify prognostic factors for the CSS (Figure 5) and OS (Figure S1) of LGBSG patients. Race, surgery, histology, and adjuvant therapy were incorporated into the nomogram for CSS (Figure 6). And metastasis, age group, histology, adjuvant therapy, and WHO grade were incorporated into the nomogram for OS (Figure S2). The calibration curve for the probability of postoperative CSS (Figure 6) and OS (Figure S2) at 5- and 8-year showed that there was a good consistency between the predicted survival probability and the actual survival probability in the data set. The C-index of internal validation for CSS and OS prediction was 0.87 (95\% CI, 0.78-0.96) and 0.78 (95\% CI, 0.70-0.86), respectively. The time-dependent ROC curve and area under curve (AUC) were established. Generally, the AUCs for CSS (Figure 7) and OS (Figure S3) at different time points in training and validation cohort were $\sim 0.7$, which suggested the nomogram was accurate and effective at different time points. The clinical use was evaluated by DCA; the 5-year DCA curves in training and validation cohort for CSS nomogram (Figure 8) and OS nomogram 


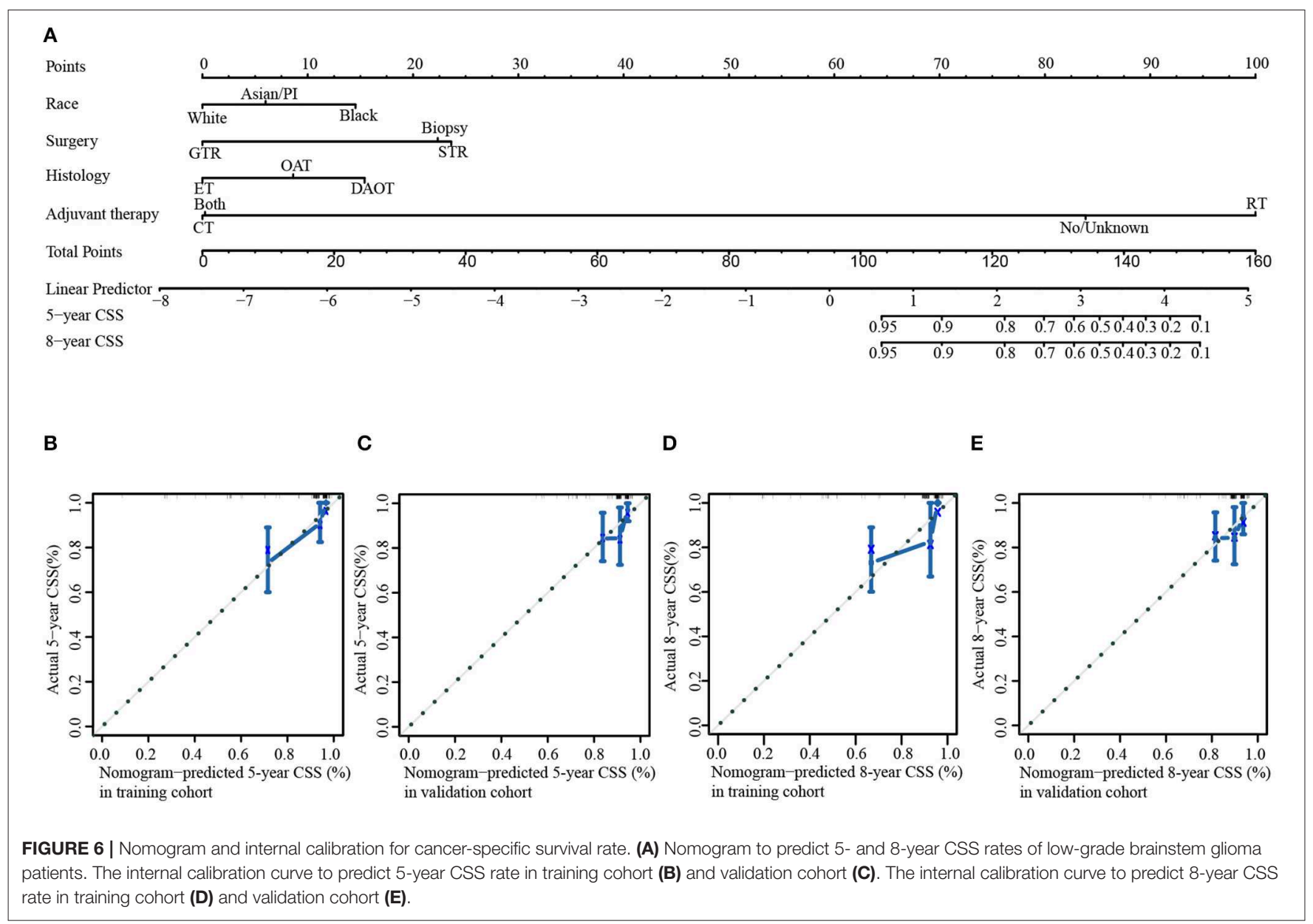

(Figure S4) yield larger net benefits than the model including surgery only.

\section{DISCUSSION}

Marked differences were observed in the epidemiological and biological characteristics of pediatric and adult patients with LGBSG. The multivariate analysis showed that the pediatric group was a significant and independent predictor of better OS and CSS. At the time of data collection, the CSS rates for pediatric and adult patients were 93.9 and $86.4 \%(P=$ 0.042), respectively. For pediatric patients with LGBSG, one retrospective study carried out at the Mayo clinic including 48 pediatric patients with LGBSG showed a median OS of 177.6 months and 5-year OS rate of 67\% (21). Another retrospective study of 52 pediatric focal LGBSG patients reported a 5-year OS rate of $98 \%$ (20). For adult LGBSG patients, Reithmeier et al. (6) reported that the median OS for 30 WHO grade II BSG patients was 26.2 months, and Kesari et al. (22) reported that the median OS for $16 \mathrm{WHO}$ grade I patients was 83 months. Generally, the results of other clinical studies support our conclusion that pediatric patients have a better survival than adult patients. In this present cohort, our analysis showed that more pediatric patients had tumors
$>3.6 \mathrm{~cm}$, and more adult patients had tumors involving the ventricular system, which had not been reported by other studies yet.

To investigate the effect of tumor characteristics on patient survival, we performed univariate and multivariate analyses and plotted Kaplan-Meier curves and decision tree. Our analysis revealed that WHO grade II was independently associated with worse CSS and OS in univariate analysis. The Kaplan-Meier curve also showed that the patients with WHO grade II tumor had worse survival rate. In this cohort, the 10-year CSS rates of patients with WHO grade I tumor and with WHO grade II tumor were 55.1 and $44.9 \%$, respectively. Consistently, Ahmed et al. (21) reported that the 5-year OS rate of patients with WHO grade I tumors was significantly higher than that of patients with WHO grade II tumors in a study with 48 pediatric LGBSG patients (71 vs. $52 \%, P=0.08$ ). In clinical studies investigating adult BSG patients, those with WHO grade I tumors exhibited better survival rate than patients WHO grade II tumor (6). In addition, we divided the histological type into DAOT, OAT, and ET according to the 2016 WHO classification of tumors of central nerves system. And data analysis showed that DAOT was an important predictor for worse survival. The 10-year CSS rate of patients with DAOT and ET were 59.1 and $88.2 \%$, respectively. This phenomenon had not been 
A

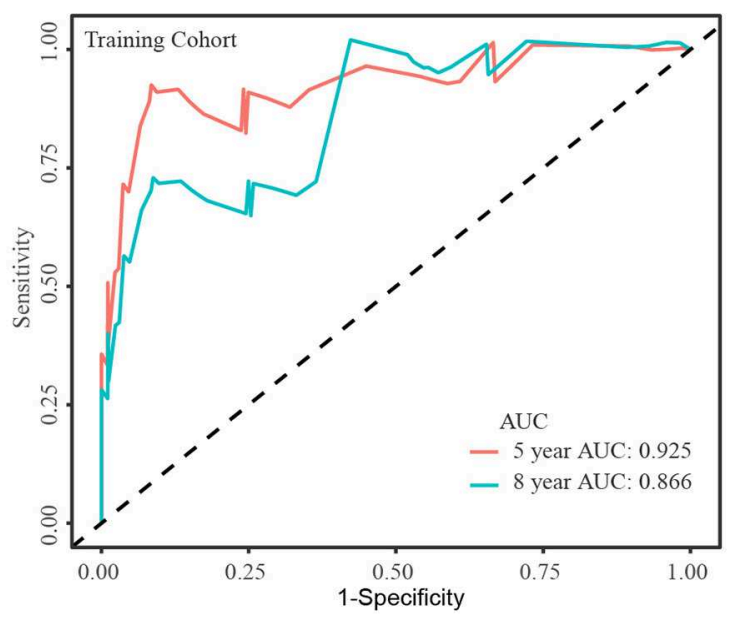

C

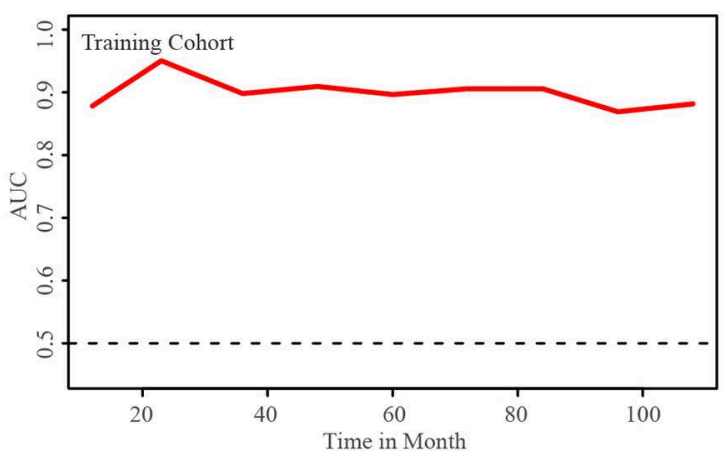

B

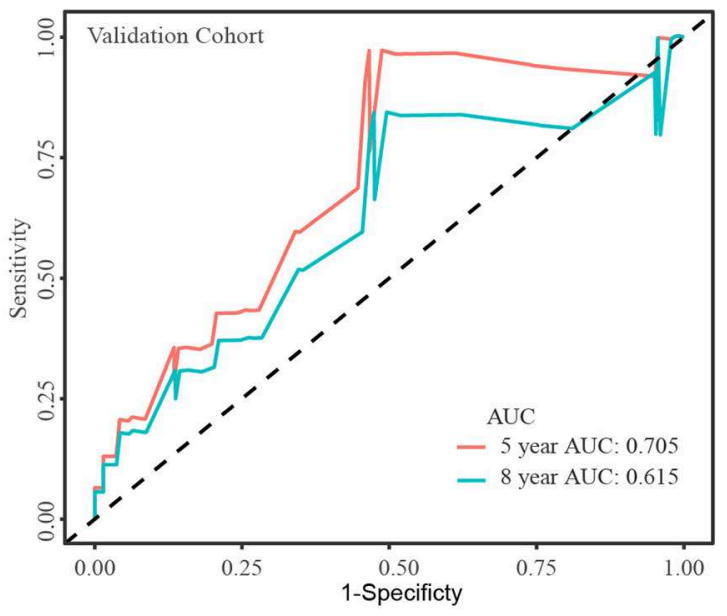

D

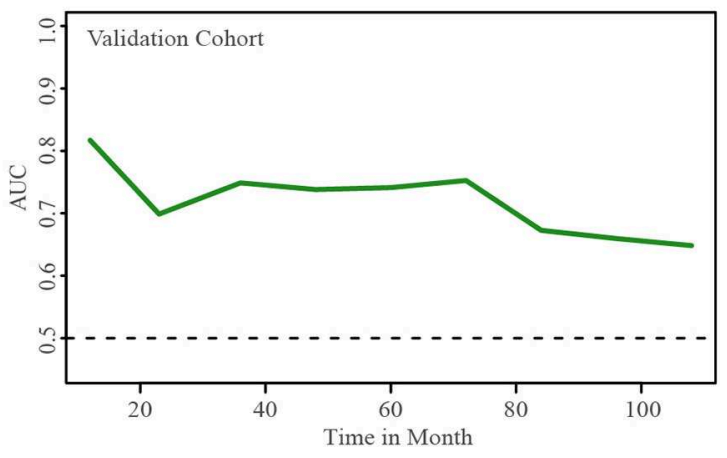

FIGURE 7 | Time-dependent ROC curve and areas under ROC curve at different time points. Areas under ROC curve of 5-and 8-year cancer-specific survival rates in training cohort (A) and validation cohort (B). Areas under ROC curve at different time points in training cohort (C) and validation cohort (D).

reported by other groups yet, which gave us a new insight about LGBSG.

Surgical treatment of the brainstem tumor has often been considered to be a difficult operation due to its critical position and crucial fundamental function. However, the advances in neuroimaging techniques (high-resolution MRI), anesthesia and neurosurgery have rendered resection of brainstem tumors feasible. Based on our analysis, GTR was significantly associated with better CSS and OS in univariate analysis when compared with patients treated with biopsy, but lost its significance in multivariate analysis. And this might be caused by the small sample size analyzed $(n=180)$ as we did not include the patients with unknown surgery status. The Kaplan-Meier curve confirmed that the patients receiving GTR had the highest survival rate $(P=0.0012)$. The 10 -year CSS rates for patients treated with GTR and biopsy were 93.1 and $81.3 \%$, respectively. So far, several studies have been reported concerning partial and even complete resection of brainstem tumor, and clinical outcomes in such studies are positive (23-25). A study conducted by Mayo Clinic on pediatric LGBSG patients concluded that tumor resection vs. biopsy only improved patient survival with statically increased 5 -year OS rate (85 vs. $50 \%, P=0.002$ )
(21). Teo and Siu (23) reported a 100\% 5-year OS rate of 23 pediatric LGBSG patients treated with endoscope-assisted microsurgery. Lundar et al. (14) performed resections on 15 pediatric patients diagnosed with low-grade midbrain glioma. They reported prolonged survival period of the patients. This study also found that tumor extension to ventricular system was a significant predictor of CSS by univariate and multivariate analyses. A population-based study focusing on HGBSG also concluded that ventricular system involvement may increase patient survival at 9 months compared with those with tumors confined to the brainstem (9). And this might be because tumors involving the ventricular system are more amenable to surgical resection. Although there is high heterogeneity that existed within different clinical studies, we could make a conclusion that most of the LGSBG patients benefited from GTR with prolonged survival. On the basis of the present analysis and results from other clinical studies (Table 4), it is considerable to suggest the safe maximal surgical resection as an effective treatment for LGBSG patients.

Gross total resection plays an important role in the management of LGBSG patients and is considered to be a favorable predictor of better CSS and OS. However, for patients 

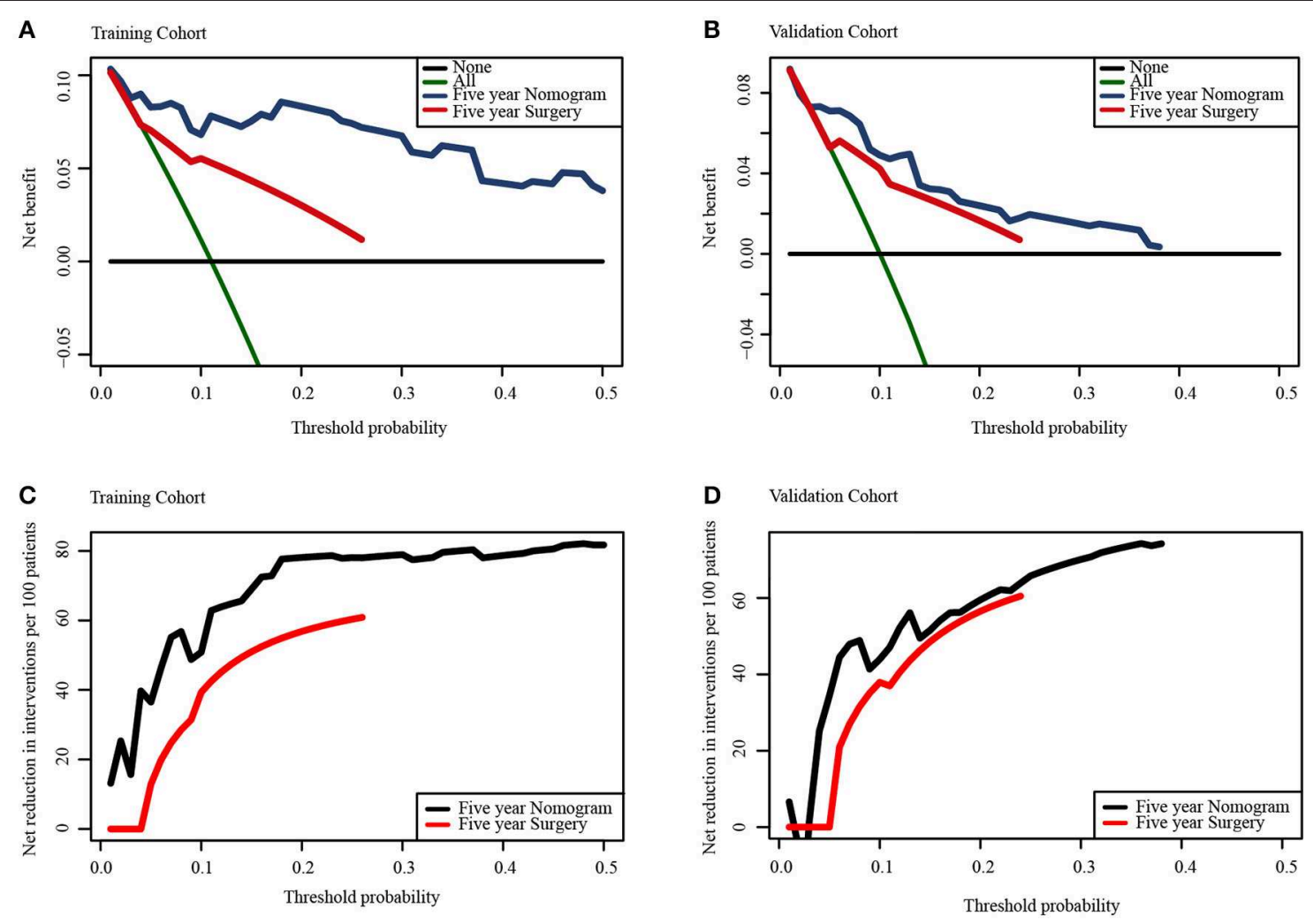

FIGURE 8 | Decision curve analysis for the nomogram and the model including surgery only in the prediction of the cancer-specific survival rates of patients at 5 -year point in training cohort $(\mathbf{A}, \mathbf{C})$ and validation cohort $(\mathbf{B}, \mathbf{D})$.

TABLE 4 | Studies reporting prognostic factors of pediatric low-grade brainstem glioma patients.

\begin{tabular}{|c|c|c|c|c|}
\hline References & Patient included & Patient number & $\begin{array}{c}\text { Median } \\
\text { follow-up (mo) }\end{array}$ & Prognostic factors \\
\hline Sandri et al. (13) & Focal BSG & 17 & 25 & GTR \\
\hline Klimo et al. (20) & Focal LGBSG & 52 & 120 & GTR, intrinsic tumor \\
\hline Lundar et al. (14) & $\begin{array}{l}\text { Low-grade } \\
\text { midbrain glioma }\end{array}$ & 15 & 96 & GTR \\
\hline Upadhyaya et al. (12) & LGBSG & 23 & 106 & $\begin{array}{l}\text { Combination of } \\
\text { surgery, RT and CT }\end{array}$ \\
\hline
\end{tabular}

undergoing STR or biopsy only with residual lesion, there is no consensus as to whether they benefit from upfront postoperative adjuvant therapy (RT/CT). Generally, our results show that more adult LGBSG patients received RT, whereas more pediatric LGBSG patients were treated with CT for adjuvant therapy. This is because that RT has been reported to cause neurocognitive deficits and academic achievement problems in pediatric patients (26). Surprisingly, the univariate analysis results showed that RT was associated with worse CSS and OS, but this effect was lost after the correction of multivariate analysis. At the same time, Kaplan-Meier curve showed that adjuvant therapy added no benefits in patients with GTR and non-GTR. Ahmed et al. (21) also concluded that postoperative RT was associated with decreased OS based on univariate analysis. Given that RT was preferentially performed in adult patients or those who received no surgery and biopsy only was an important reason, this selection bias may account for this effect. Furthermore, univariate analysis showed that CT was not significantly associated with CSS and OS. Indeed, no CT regimen has been proven to be effective for LGBSG patients. Only few cases were reported to 
have a good response to CT $(14,27,28)$. In addition, the KaplanMeier curves plotted both in all patients $(n=305)$ and nonGTR group ( $n=125$ ) supported that adjuvant therapy provided no clinical benefits. A clinical study including 96 pediatric patients with LGBSG reported that upfront adjuvant therapy (RT/CT) did not significantly improve the prognosis of patients with residual tumor compared with observation only (15). In summary, upfront adjuvant therapy is not beneficial to CSS and OS. Therefore, observation may be a safe alternative for LGBSG patients receiving STR or biopsy only with residual lesion.

This study has the following limitations. Although the SEER database contains a large number of records, it lacks other important information. For instance, data about the concrete position and growth pattern of tumor were not accessible in SEER database, which were important for tumor categorization and evaluating the prognosis of patients with LGBSG $(20,21,29)$. Moreover, other clinical features of patients including functional status and neurologic symptoms were not available, which were reported to be predictive factors of patient prognosis (30-32). In addition, the surgical approach, radiation dosage, and CT protocol were not included in our analysis, although these factors influence the patient prognosis. And we do not know the detail information about how the tumor size was measured. Another weakness of this study is that there are 61 patients with specific histological diagnosis having the record of "no surgery" in the SEER database, which is confusing. So we did not include these 61 patients in the data set so as to make reliable conclusion. Given that the surgical experience is growing and efforts are made to attempt preferable adjuvant therapy methods (16, 33-35), the present study based on cases reported between 2004 and 2015 could not capture the most updated clinical evidence.

The strengths of this study include being the largest population-based real world study about LGBSG from SEER database. This study confirmed many findings in other single-center small dataset clinical studies with increased strength and low bias. This study describes, for the first time, the characteristics and prognostic factors of adult patients with LGBSG and compared the differences between pediatric and adult patients with LGBSG. Moreover, an accurate and effective nomogram was established to predict CSS and OS rate of LGBSG patients.

\section{CONCLUSION}

This population-based real world study of 165 pediatric and 140 adult LGBSG patients demonstrates that there are differences

\section{REFERENCES}

1. Ostrom QT, Gittleman H, Liao P, Vecchione-Koval T, Wolinsky Y, Kruchko $\mathrm{C}$, et al. CBTRUS statistical report: primary brain and other central nervous system tumors diagnosed in the united states in 2010-2014. Neuro Oncol. (2017) 19(Suppl. 5):v1-88. doi: 10.1093/neuonc/nox158

2. Hu J, Western S, Kesari S. Brainstem glioma in adults. Front Oncol. (2016) 6:180. doi: $10.3389 /$ fonc. 2016.00180 between these two groups. And safe maximal surgical resection was suggested as an effective treatment according to our data analysis and other clinical studies, yet with caution surgical resection may result in significant neurological deficits. Observation seems to be optional for patients with residual tumor after incomplete surgical resection because upfront adjuvant therapy had no effect on CSS according to the analysis. This study provides valuable data highlighting the need for prospective clinical studies in order to validate outcomes.

\section{DATA AVAILABILITY STATEMENT}

The data of LGBSG patients searched in SEER database are freely available.

\section{AUTHOR CONTRIBUTIONS}

QC and SF made substantial contribution to the design of this study. ZhuL and QC carried out the analysis and interpreted the data. ZhuL and SF made contributions to the drafting of the manuscript. JL and HC made contributions to the review of previous literature. JH, FF, and LC contributed substantially to the revision of the manuscript. QC and ZhiL made substantial contributions to the conception of the manuscript, and were responsible for the quality of the overall manuscript. All authors approved the final version of the manuscript.

\section{FUNDING}

This work was supported by the National Natural Science Foundation of China (Nos. 81703622, 81472693, and 81873635), China Postdoctoral Science Foundation (No. 2018M633002), Hunan Provincial Natural Science Foundation of China (No. 2018JJ3838), Hunan provincial health and Health Committee Foundation of China (C2019186).

\section{ACKNOWLEDGMENTS}

The authors acknowledge the efforts of the SEER Program cancer registries in creating the SEER database.

\section{SUPPLEMENTARY MATERIAL}

The Supplementary Material for this article can be found online at: https://www.frontiersin.org/articles/10.3389/fonc. 2020.00391/full\#supplementary-material

3. Choux M, Lena G, Do L. Brainstem tumors. In: Choux M, Di Rocco C, Hockley A, Walker M editors. Pediatric Neurosurgery. London: Churchill Livingstone (1999). p. 471-89.

4. Hargrave D, Bartels $U$, Bouffet E. Diffuse brainstem glioma in children: critical review of clinical trials. Lancet Oncol. (2006) 7:241-8. doi: 10.1016/S1470-2045(06)70615-5

5. Reyes-Botero G, Laigle-Donadey F, Mokhtari K, Martin-Duverneuil N, Delattre JY. Temozolomide after radiotherapy in recurrent "low grade" 
diffuse brainstem glioma in adults. J Neuro Oncol. (2014) 120:5816. doi: 10.1007/s11060-014-1589-9

6. Reithmeier T, Kuzeawu A, Hentschel B, Loeffler M, Trippel M, Nikkhah G. Retrospective analysis of 104 histologically proven adult brainstem gliomas: clinical symptoms, therapeutic approaches and prognostic factors. BMC Cancer. (2014) 14:115. doi: 10.1186/1471-2407-14-115

7. Khalid SI, Kelly R, Adogwa O, Carlton A, Tam E, Naqvi S, et al. Pediatric brainstem gliomas: a retrospective study of 180 patients from the seer database. Pediatr Neurosurg. (2019) 54:151-164. doi: 10.1159/000497440

8. Wu G, Broniscer A, McEachron TA, Lu C, Paugh BS, Becksfort J, et al. Somatic histone $\mathrm{H} 3$ alterations in pediatric diffuse intrinsic pontine gliomas and nonbrainstem glioblastomas. Nat Gene. (2012) 44:251-3. doi: 10.1038/ng.1102

9. Maxwell R, Luksik AS, Garzon-Muvdi T, Yang W, Huang J, Bettegowda C, et al. Population-based study determining predictors of cancer-specific mortality and survival in pediatric high-grade brainstem glioma. World Neurosurg. (2018) 119:e1006-e15. doi: 10.1016/j.wneu.2018.08.044

10. Brat DJ, Verhaak RG, Aldape KD, Yung WK, Salama SR, Cooper LA, et al. Comprehensive, integrative genomic analysis of diffuse lower-grade gliomas. N Eng J Med. (2015) 372:2481-98. doi: 10.1056/NEJMoa1402121

11. Simonetti G, Gaviani P, Botturi A, Innocenti A, Lamperti E, Silvani A. Clinical management of grade III oligodendroglioma. Cancer Manag Res. (2015) 7:213-23. doi: 10.2147/CMAR.S56975

12. Upadhyaya SA, Koschmann C, Muraszko K, Venneti S, Garton HJ, Hamstra DA, et al. Brainstem low-grade gliomas in children-excellent outcomes with multimodality therapy. J Child Neurol. (2017) 32:194203. doi: $10.1177 / 0883073816675547$

13. Sandri A, Sardi N, Genitori L, Giordano F, Peretta P, Basso ME, et al. Diffuse and focal brain stem tumors in childhood: prognostic factors and surgical outcome. Experience in a single institution. Childs Nerv Syst. (2006) 22:1127-35. doi: 10.1007/s00381-006-0083-x

14. Lundar T, Due-Tonnessen BJ, Egge A, Scheie D, Brandal P, Stensvold E, et al. Neurosurgical treatment of pediatric low-grade midbrain tumors: a single consecutive institutional series of 15 patients. J Neurosurg Pediatrics. (2014) 14:598-603. doi: 10.3171/2014.9.PEDS1462

15. Fried I, Hawkins C, Scheinemann K, Tsangaris E, Hesselson L, Bartels $\mathrm{U}$, et al. Favorable outcome with conservative treatment for children with low grade brainstem tumors. Pediatr Blood Cancer. (2012) 58:55660. doi: $10.1002 / p b c .23200$

16. Ronghe M, Hargrave D, Bartels U, Tabori U, Vaidya S, Chandler C, et al. Vincristine and carboplatin chemotherapy for unresectable and/or recurrent low-grade astrocytoma of the brainstem. Pediatr Blood Cancer. (2010) 55:4717. doi: $10.1002 / \mathrm{pbc} .22557$

17. Mundinger F, Braus DF, Krauss JK, Birg W. Long-term outcome of 89 low-grade brain-stem gliomas after interstitial radiation therapy. J Neurosurg. (1991) 75:740-6. doi: 10.3171/jns.1991.75. 5.0740

18. Liao CH, Pan DH, Yang HC, Wu HM, Ho DM, Wong TT, et al. Gamma Knife radiosurgery as a treatment modality for low-grade pediatric brainstem gliomas: report of two cases. Child Nerv Syst. (2012) 28:1758. doi: 10.1007/s00381-011-1620-9

19. Yang Z, Zhang Z, Wang X, Hu Y, Lyu Z, Huo L, et al. Intensity-modulated radiotherapy for gliomas:dosimetric effects of changes in gross tumor volume on organs at risk and healthy brain tissue. Oncotargets Ther. (2016) 9:354554. doi: $10.2147 / O T T . S 100455$

20. Klimo P Jr, Pai Panandiker AS, Thompson CJ, Boop FA, Qaddoumi I, Gajjar A, et al. Management and outcome of focal low-grade brainstem tumors in pediatric patients: the St. Jude experience. J Neurosurg Pediatr. (2013) 11:274-81. doi: 10.3171/2012.11.PEDS12317

21. Ahmed KA, Laack NN, Eckel LJ, Orme NM, Wetjen NM. Histologically proven, low-grade brainstem gliomas in children: 30-year experience with long-term follow-up at mayo clinic. Am J Clin Oncol. (2014) 37:516. doi: $10.1097 / C O C .0 b 013 e 31826 b 9903$

22. Kesari S, Kim RS, Markos V, Drappatz J, Wen PY, Pruitt AA. Prognostic factors in adult brainstem gliomas: a multicenter, retrospective analysis of 101 cases. J Neuro Oncol. (2008) 88:175-83. doi: 10.1007/s11060-008-9545-1

23. Teo C, Siu TL. Radical resection of focal brainstem gliomas: is it worth doing? Childs Nerv Syst. (2008) 24:1307-14. doi: 10.1007/s00381-008-0647-z

24. Lesniak MS, Klem JM, Weingart J, Carson BS Sr. Surgical outcome following resection of contrast-enhanced pediatric brainstem gliomas. Pediatr Neurosurg. (2003) 39:314-22. doi: 10.1159/000075260

25. Watanabe $\mathrm{T}$, Sato $\mathrm{T}$, Kishida $\mathrm{Y}$, Ito $\mathrm{E}$, Ichikawa $\mathrm{M}$, Sakuma J, et al. Endoscopic resection of cystic pontine tumour: three case reports and a proposal for minimally invasive dual-endoscopic surgery. Acta Neurochir. (2014) 156:1145-50. doi: 10.1007/s00701-014-2085-x

26. Willard VW, Conklin HM, Boop FA, Wu S, Merchant TE. Emotional and behavioral functioning after conformal radiation therapy for pediatric ependymoma. Int J Radiat Oncol Biol Phys. (2014) 88:814-21. doi: 10.1016/j.ijrobp.2013.12.006

27. Wu J, Neale N, Huang Y, Bai HX, Li X, Zhang Z, et al. Comparison of adjuvant radiation therapy alone and chemotherapy alone in surgically resected lowgrade gliomas: survival analyses of 2253 cases from the national cancer data base. World Neurosurg. (2018) 112:e812-22. doi: 10.1016/j.wneu.2018.01.163

28. Hong J, Peng Y, Liao Y, Jiang W, Wei R, Huo L, et al. Nimotuzumab prolongs survival in patients with malignant gliomas: a phase I/II clinical study of concomitant radiochemotherapy with or without nimotuzumab. Exp Ther Med. (2012) 4:151-7. doi: 10.3892/etm.2012.555

29. He Y, Wang Y, Shen L, Zhao Y, Cao P, Lei M, et al. Prognostic value of the distance between the primary tumor and brainstem in the patients with locally advanced nasopharyngeal carcinoma. BMC Cancer. (2016) 16:114. doi: 10.1186/s12885-016-2148-x

30. Ueoka DI, Nogueira J, Campos JC, Maranhao Filho P, Ferman S, Lima MA. Brainstem gliomas-retrospective analysis of 86 patients. J Neurol Sci. (2009) 281:20-3. doi: 10.1016/j.jns.2009.03.009

31. Sun T, Wan W, Wu Z, Zhang J, Zhang L. Clinical outcomes and natural history of pediatric brainstem tumors: with 33 cases follow-ups. Neurosurg Rev. (2013) 36:311-9. doi: 10.1007/s10143-012-0428-8

32. Jiang T, Mao Y, Ma W, Mao Q, You Y, Yang X, et al. CGCG clinical practice guidelines for the management of adult diffuse gliomas. Cancer Lett. (2016) 375:263-73. doi: 10.1016/j.canlet.2016.01.024

33. Phuong PC, Hung NQ, Ngoc TB, Rades D, Khoa MT. Rotating gamma system irradiation: a promising treatment for low-grade brainstem gliomas. In Vivo. (2017) 31:957-60. doi: 10.21873/invivo.11153

34. El-Shehaby AM, Reda WA, Abdel Karim KM, Emad Eldin RM, Esene IN. Gamma knife radiosurgery for low-grade tectal gliomas. Acta Neurochir. (2015) 157:247-56. doi: 10.1007/s00701-014-2299-y

35. Mottolese C, Szathmari A, Beuriat PA, Frappaz D, Jouvet A, Hermier M. Tectal plate tumours. Our experience with a paediatric surgical series. Neuro Chirurgie. (2015) 61:193-200. doi: 10.1016/j.neuchi.2013.12.007

Conflict of Interest: The authors declare that the research was conducted in the absence of any commercial or financial relationships that could be construed as a potential conflict of interest.

Copyright (c) 2020 Liu, Feng, Li, Cao, Huang, Fan, Cheng, Liu and Cheng. This is an open-access article distributed under the terms of the Creative Commons Attribution License (CC BY). The use, distribution or reproduction in other forums is permitted, provided the original author(s) and the copyright owner(s) are credited and that the original publication in this journal is cited, in accordance with accepted academic practice. No use, distribution or reproduction is permitted which does not comply with these terms. 